\title{
UNIQUE CONTINUATION WITH WEAK TYPE LOWER ORDER TERMS: THE VARIABLE COEFFICIENT CASE
}

\author{
Guozhen Lu ${ }^{1}$
}

\begin{abstract}
This paper deals with the unique continuation problems for variable coefficient elliptic differential equations of second order. We will prove that the unique continuation property holds when the variable coefficients of the leading term are Lipschitz continuous and the coefficients of the lower order terms have small weak type Lorentz norms. This will improve an earlier result of T. Wolff in this direction.
\end{abstract}

\section{Introduction}

Unique continuation problems for variable coefficient elliptic differential equations of second order have been studied by many authors. We refer the reader to $[\mathbf{7}],[\mathbf{8}]$ and $[\mathbf{9}]$ for the most recent results and many references therein.

The following result we prove here is an improvement of a theorem in $[\mathbf{9}]$.

Theorem 1. If $d \geq 3$, then there is a constant $\epsilon=\epsilon_{d, \lambda}>0$ making the following true. Assume that $\Omega \subset \mathbb{R}^{d}$ is a domain and $L=$ $\sum_{i, j=1}^{d} a_{i j}(x) \frac{\partial^{2}}{\partial x_{i} \partial x_{j}}$ is an elliptic operator with Lipschitz coefficients on $\Omega, A: \Omega \rightarrow \mathbb{R}$ and $B: \Omega \rightarrow \mathbb{R}$ are functions such that (1.1)-(1.3) below hold:

$$
\begin{aligned}
& \lim _{r \rightarrow 0}\|A\|_{L^{p \infty}(D(a, r))} \leq \epsilon_{d} \\
& \lim _{r \rightarrow 0}\|B\|_{L^{d \infty}(D(a, r))} \leq \epsilon_{d}
\end{aligned}
$$

${ }^{1}$ The author is supported in part by NSF Grant \#DMS93-15963. AMS classification 35,42 . 


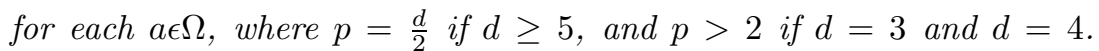
Assume also that $u \in W_{\mathrm{loc}}^{22}(\Omega)$ and satisfies

$$
|L u| \leq A|u|+B|\nabla u| .
$$

Then if $u$ vanishes on an open set it vanishes identically.

In the above, "elliptic" means that the matrix $a_{i j}(x)$ is real and positive definite for each $x \in \Omega$, i.e., there exists a positive constant $\lambda$ such that

$$
\lambda^{-1}|\xi|^{2} \leq \sum_{i, j=1}^{d} a_{i j}(x) \xi_{i} \xi_{j} \leq \lambda|\xi|^{2}
$$

for each $x \in \Omega$ and $\xi=\left(\xi_{j}\right)_{j=1}^{d} \in R^{d}$; $W^{22}$ is the Sobolev space, i.e., functions whose second derivatives are in $L^{2} ;\|A\|_{L^{q \infty}(D(a, r))}$ is the weak type norm defined as follows:

$$
\|A\|_{L^{q \infty}(D(a, r))}=\sup _{\lambda>0}\left(\lambda^{q}|\{x \in D(a, r):|A(x)|>\lambda\}|\right)^{1 / q} .
$$

This theorem is a refinement of the previous result of [9], in which the theorem was shown to be true for $A \in L_{\text {loc }}^{p}$ and $B \in L_{\text {loc }}^{d}$, and is an extension to the variable coefficient case of the result in $[\mathbf{3}]$. Such type of extension to weak type zero order case was considered earlier in $[\mathbf{6}]$, which sharpens the result of $[\mathbf{2}]$. It is known that the Lipschitz condition can not be replaced by any weaker Holder condition (see [4]). For the previous known results for unique continuation in this direction, we refer the reader to $[\mathbf{7}]$ and $[\mathbf{8}]$, where the unique continuation and strong unique continuation when $B=0$ and $A \in L_{\text {loc }}^{\frac{d}{2}}$ for $a_{i j}(x) \in C^{\infty}$ were proved. For an alternate way of sharpening the result with $L^{p}$ potential by using Campanato-Morrey type condition, we refer the reader to $[\mathbf{1}],[\mathbf{5}]$, and $[\mathbf{1 0}]$.

We will use the covering lemma (Lemma 1') of [9] and the method of freezing coefficients on appropriate convex and compact sets to prove our theorem. We will adapt the idea of bounding the weak $L^{d}$ norm of $B$ from [3]. However, unlike the constant coefficient case (see $[\mathbf{3}]$ ), we will not be able to control the weak $L^{d}$ norm of $B$ alone when we deal with the variable coefficient case because we can not drop the terms containing the weak $L^{p}$ norm of $A$ due to the presence of other quantities (see Section 3 for more details). Thus, in contrast to [3], in order to prove Theorem 1 we shall show either the weak $L^{p}$ ( $p$ is as in Theorem 1) norm of the zero order term $A$ or the weak $L^{d}$ norm of the lower order term $B$ over the union of some (many enough) disjoint sets would not be too 
small if the unique continuation property failed. In any case, it would lead to a contradiction and the theorem follows.

Section 2 of this paper is the Carleman inequalities needed for Theorem 1, and Section 3 is the proof of the theorem.

One word about the notation: we always assume $d \geq 3$ in this paper; constants depend on the dimension $d$ and the elliptic constant $\lambda$ only unless otherwise specified and may differ from lines to lines; we write $x \preceq y$ to mean $x \leq C y$ and $x \approx y$ for $x \preceq y$ and $y \preceq x$.

Acknowledgement. I am greatly indebted to T. Wolff for his encouragement and helpful discussions.

\section{Carleman type of inequalities}

Hereafter, $f^{\wedge}$ and $f^{v}$ denote the Fourier and inverse Fourier transforms respectively. We now define the multiplier $N_{k}$ by $\left(e^{k \cdot x} u\right)^{\wedge}=N_{k}\left(e^{k \cdot x} \triangle\right.$ $u)^{\wedge}$, where

$$
N_{k}(\xi)=\frac{1}{|\xi|^{2}-i k \cdot \xi-|k|^{2}}
$$

Take $\phi \in C_{0}^{\infty}(D(0,2))$, and $\phi=1$ on $D(0,1)$, where $D(0, a)=\{x:|x|<$ $a\}$. Set $\phi^{(k)}(x)=\phi(|k| x)$. Then we can define three multiplier operators, $T, T_{1}$, and $T_{2}$ by

$$
\widehat{T f}=N_{k} \hat{f}, \widehat{T_{1} f}=N_{k}\left(1-\phi_{k}\right) \hat{f}, \widehat{T_{2} f}=\left(N_{k} \cdot \phi_{k}\right) \hat{f} .
$$

Thus we have the following lemma

Lemma 2.1. Suppose $k \in R^{d}, E \subset R^{d}$ compact and convex, $|E| \geq$ $|k|^{-d}, u \in W^{22}$ has compact support, then if

i) Suppose either a) $d \geq 5$ and $\theta>\frac{d-4}{d(d-1)}, \frac{1}{2}-\frac{1}{q}=\frac{1}{t} \geq \frac{2}{d}$; or b) $d=3$ or $d=4,0<\theta<\frac{1}{d+1}$ and $\frac{1}{2}-\frac{1}{q}=\frac{1}{t}>\frac{1}{2}-\frac{\theta(d-1)}{2} ;$ The following holds:

$$
\left\|T_{2}\left(e^{k \cdot x} \triangle u\right)\right\|_{q} \leq C_{\theta}^{-1}|k|^{\frac{d}{t}-2}\left(|k|^{d}|E|\right)^{\theta}\left\|e^{k \cdot x} \triangle u\right\|_{2, E} .
$$

ii) Suppose either a) $d \geq 5, \frac{1}{2}-\frac{1}{q}=\frac{2}{d}$ and $\theta>\frac{d-4}{d(d-1)}$, or b) $d=4$, $\frac{2}{d}>\frac{1}{2}-\frac{1}{q}>\frac{1}{2}-\frac{\theta(d-1)}{2}$ and $0<\theta<\frac{1}{d+1}$; or $\left.c\right) d=3, \frac{1}{2}-\frac{1}{q}>$ $\frac{1}{2}-\frac{\theta(d-1)}{2}$ and $0<\theta<\frac{1}{d+1}$. If $d=3$ or 4 , assume $|k| \geq 1$. Then

$$
\left\|e^{k \cdot x} u\right\|_{q 2} \leq C_{\theta}^{-1}\left(|k|^{d}|E|\right)^{\theta}\left\|e^{k \cdot x} \triangle u\right\|_{2, E} .
$$


We note in the above that $\|\cdot\|_{q p}$ is the Lorentz norm defined by $\|f\|_{q p}=\left(q \int_{0}^{\infty} s^{p-1}|\{x:|f(x)|>s\}|^{p / q} d s\right)^{\frac{1}{p}}$ and $\|f\|_{2, E}=\left\|f g_{E}\right\|_{2}$ where $g_{E}$ is defined for the compact and convex set $E$ by $g_{E}(x)=$ $\min \{T \geq 1: x \in T E\}$ where $T E$ is the expansion of $E$ around the barycenter of $E$.

Remark. 1) We note the values of $\theta>\theta_{d}=\max \left(\frac{d-4}{d(d-1)}, 0\right)$ in Lemma (2.1) is sharper than those in the similar type of inequalities in [9] (see Lemma 6.4 in [9]). However, it is of no essential use for the present purpose. As long as we can take $\theta<\frac{1}{d}$, it will be good enough in the later argument in Section 3.

2) In the above lemma (ii) if we set $\frac{1}{2}-\frac{1}{q}=\frac{1}{p}$, then we can certainly take $p=\frac{d}{2}$ if $d \geq 5 ; p>2$ if $d=4$ ( $\theta$ can be as small as possible provided that $p>2$ is very close to 2); and $p>2$ and any $\theta>0$ if $d=3$ (actually $p=2$ is allowed). In (i) of the lemma, we can take $t<p$ for the corresponding $p$ we just mentioned.

3) We note we may assume $k=e_{1}$ in proving lemma (2.1), since inequalities (i) and (ii) a) are scale invariant and in (ii) b) and c), the scaling works for $|k|$ large.

Proof of Lemma (2.1):

Let $N(\xi)=\frac{1}{|\xi|^{2}-i \xi_{1}-1}=N_{e_{1}}(\xi)$. Let also $N_{1}(\xi)=N(\xi) \cdot(1-\phi)(\xi)$ and $N_{2}(\xi)=N(\xi) \phi(\xi)$, where $\phi(\xi)=\phi^{\left(e_{1}\right)}(\xi)$ as before. Then

$$
\left|N_{1}(\xi)\right| \leq\left(1+|\xi|^{2}\right)^{-1}
$$

It is shown in Lemma 6.3 of $[\mathbf{9}]$ that

$$
\left\|\left(N_{2} \hat{f}\right)^{v}\right\|_{q_{1}} \leq C\|f\|_{p_{1}}
$$

provided

$$
1 \leq p_{1} \leq s, \frac{1}{q_{1}}<\frac{1}{p_{1}^{\prime}}+\frac{1}{2}-\frac{s^{\prime}}{2 p_{1}^{\prime}},
$$

where $s=\frac{2 d+2}{d+3}$ is the Stein-Tomas exponent in the restriction theorem for the Fourier transform, and $p^{\prime}$ denotes the conjugate of $p$.

By duality,

$$
\left\|\left(N_{2} \hat{f}\right)^{v}\right\|_{p_{1}^{\prime}} \leq C\|f\|_{q_{1}^{\prime}}
$$


We note $(2.3)$ is equivalent to

$$
\frac{1}{q_{1}^{\prime}}>\frac{1}{2}+\frac{1}{p_{1}^{\prime}} \frac{2}{d-1} \text { and } 1 \leq p_{1} \leq s .
$$

We now let $p_{1}=\frac{2 d}{d+4}$ (which is less than $s$ ) and $\theta>\frac{d-4}{d(d-1)}$ when $d \geq 5$. Then by (2.5), once we select $q_{1}$ such that $\frac{1}{p_{1}^{\prime}} \frac{2}{d-1}<\frac{1}{q_{1}^{\prime}}-\frac{1}{2}<\theta$, for such $q_{1}$ and $p_{1}(2.4)$ holds.

Since $N_{2}(\xi)$ has compact support, then for any $q \geq p_{1}^{\prime}$ we have

$$
\begin{aligned}
\left\|\left(N_{2} \hat{f}\right)^{v}\right\|_{q} \leq C\left\|\left(N_{2} \hat{f}\right)^{v}\right\|_{p_{1}^{\prime}} \leq C\|f\|_{q_{1}^{\prime}} \\
\quad \leq C|E|^{\frac{1}{q_{1}^{\prime}}-\frac{1}{2}}\|f\|_{2, E} \leq C|E|^{\theta}\|f\|_{2, E} .
\end{aligned}
$$

But $\frac{1}{2}-\frac{1}{q} \geq \frac{2}{d}$ is equivalent to $q \geq p_{1}^{\prime}$, we are then done for the case $d \geq 5$ by scaling.

When $d=3$ or $d=4$, we let $0<\theta<\frac{1}{d+1}$ and $q$ satisfy $\frac{1}{2}-\frac{1}{q}=$ $\frac{1}{t}>\frac{1}{2}-\frac{\theta(d-1)}{2}$. Thus $\frac{1}{q}<\frac{\theta(d-1)}{2}$. We can then pick $p_{1}$ such that $\frac{1}{q} \leq \frac{1}{p_{1}^{\prime}}<\frac{\theta(d-1)}{2}$ because $\frac{1}{p_{1}^{\prime}}$ is increasing as $p_{1}$ does and is at most $\frac{1}{s^{\prime}}=\frac{d-1}{2 d+2}$. Consequently we can pick $q_{1}$ such that $\frac{1}{p_{1}^{\prime}} \frac{2}{d-1}<\frac{1}{q_{1}^{\prime}}-\frac{1}{2}<\theta$. Since $q \geq p_{1}$ and $N_{2}$ has compact support, we will get

$$
\left\|\left(N_{2} \hat{f}\right)^{v}\right\|_{q} \leq C\left\|\left(N_{2} \hat{f}\right)^{v}\right\|_{p_{1}^{\prime}} \leq C|| f\left\|_{q_{1}^{\prime}} \leq C|E|^{\theta}\right\| f \|_{2, E} .
$$

This proves (i) of Lemma (2.1).

We now give the proof of (ii) of lemma (2.1). By (2.2), $N_{1}(\xi)$ is a Bessel potential of order 2. Thus for any $q$ satisfying the assumption of Lemma (2.1) (ii) we have

$$
\left\|\left(N_{1} \hat{f}\right)^{v}\right\|_{q} \leq C\|f\|_{2}
$$

which of course leads to the desired conclusion by combining with the estimate for $\left\|\left(N_{2} \hat{f}\right)^{v}\right\|_{q}$ obtained in part (i).

We now fix $p_{0}=\frac{2 d}{d+2}$ and use $p_{0}^{\prime}=\frac{2 d}{d-2}$ to express its conjugate. We assume $m_{k}(\xi)=\frac{i \xi-k}{|\xi|^{2}-i \xi k-|k|^{2}}$ for $k \in R^{d}$, i.e., a multiplier such that

$$
\left(e^{k \cdot x} \nabla u\right)^{\wedge}(\xi)=m_{k}(\xi)\left(e^{k \cdot x} \triangle u\right)^{\wedge}(\xi)
$$

for $u \in C_{0}^{\infty}\left(R^{d}\right)$, where $f^{\wedge}$ is the Fourier transform of $f$. Set $\phi^{(k)}(x)=$ $\phi(|k| x)$ as before.

We now introduce the notations of three multiplier operators

$$
\widehat{S f}=m_{k} \hat{f}, \widehat{S_{1} f}=m_{k}\left(1-\phi_{k}\right) \hat{f}, \widehat{S_{2} f}=m_{k} \phi_{k} \hat{f} \text {. }
$$

Thus

$$
e^{k \cdot x} \nabla u=S\left(e^{k \cdot x} \triangle u\right)=S_{1}\left(e^{k \cdot x} \triangle u\right)+S_{2}\left(e^{k \cdot x} \triangle u\right) .
$$

We have the following lemma: 
Lemma 2.7. If $k \in R^{d}, E \subset R^{d}$ compact and convex set, $|E| \geq$ $|k|^{-d}, u \in W^{22}$ has compact support. Then for any $\theta>\frac{d-2}{d(d-1)}$, the following two inequalities hold:

i) $\left\|S_{2}\left(e^{k \cdot x} \triangle u\right)\right\|_{q} \leq C|k|^{\frac{d}{s}-1}\left(|k|^{d}|E|\right)^{\theta}\left\|e^{k \cdot x} \triangle u\right\|_{2, E}$ provided $\frac{1}{d} \leq$ $\frac{1}{2}-\frac{1}{q}=\frac{1}{s}$

ii) $\left\|e^{k \cdot x} \nabla u\right\|_{p_{0}^{\prime} 2} \leq C\left(|k|^{d}|E|\right)^{\theta}\left\|e^{k \cdot x} \triangle u\right\|_{2, E}$.

We will prove Lemma (2.7) in the case $k=e_{1}$ and then by the scaling property the general case will follow. We need a closer look of the proof of Lemma 6.4 in $[\mathbf{9}]$.

Proof: Recall, when $k=e_{1}, m_{e_{1}}(\xi)=m(\xi)=\frac{i \xi-e_{1}}{|\xi|^{2}-i \xi_{1}-1}$. Let $m_{1}(\xi)=$ $(1-\phi(\xi)) m(\xi), m_{2}(\xi)=\phi(\xi) m(\xi)$, where $\phi(\xi)=\phi^{e_{1}}(\xi)$. Then clearly,

$$
\left|m_{1}(\xi)\right| \leq C\left(1+|\xi|^{2}\right)^{-1 / 2} \text {. }
$$

It is shown in Lemma 6.4 of $[\mathbf{9}]$ that

$$
\left\|\left(m_{2} \hat{f}\right)^{v}\right\|_{p_{0}^{\prime}} \leq C\|f\|_{r}
$$

provided $\frac{1}{r}>\frac{1}{2}+\frac{d-2}{d(d-1)}$, where $p_{0}^{\prime}=\frac{2 d}{d-2}$.

Since $m_{2}$ has compact support, then for any $q \geq p_{0}^{\prime}$,

$$
\left\|\left(m_{2} \hat{f}\right)^{v}\right\|_{q} \leq C\left\|\left(m_{2} \hat{f}\right)^{v}\right\|_{p_{0}^{\prime}} \leq C\|f\|_{r} .
$$

Note $\frac{1}{2}-\frac{1}{p_{0}^{\prime}}=\frac{1}{d}$, thus $\frac{1}{2}-\frac{1}{q} \geq \frac{1}{d}$ is equivalent to $q \geq p_{0}^{\prime}$.

Given any $\theta>\frac{(d-2)}{d(d-1)}$, we can select $r$ such that $\frac{1}{2}+\frac{d-2}{d(d-1)}<\frac{1}{r}<\frac{1}{2}+\theta$. Thus, for so selected $r,(2.9)$ holds. So for any $q$ satisfying $\frac{1}{2}-\frac{1}{q} \geq \frac{1}{d}$

$$
\left\|\left(m_{2} \hat{f}\right)^{v}\right\|_{q} \leq C\|f\|_{r} \leq C|E|^{\theta}\|f\|_{2, E} .
$$

Thus part (i) of lemma (2.7) will follow by scaling.

We now prove (ii) of Lemma (2.7). We also assume $k=e_{1}$ first. Then

$$
\left\|\left(m_{1} \hat{f}\right)^{v}\right\|_{p_{0}^{\prime} 2} \leq C\|f\|_{2}
$$

since $\left|m_{1}(\xi)\right| \leq\left(1+|\xi|^{2}\right)^{-1 / 2}$. Since the proof in $[\mathbf{9}]$ will apply to the weak type version, thus

$$
\left\|\left(m_{2} f^{\wedge}\right)^{v}\right\|_{p_{0}^{\prime} 2} \leq C\|f\|_{r 2}
$$

if $\frac{1}{2}+\theta>\frac{1}{r}>\frac{1}{2}+\frac{d-2}{d(d-1)}$. So,

$$
\left\|\left(m_{2} \hat{f}\right)^{v}\right\|_{p_{0}^{\prime} 2} \leq C|E|^{\frac{1}{r}-\frac{1}{2}}\|f\|_{2, E} \leq C|E|^{\theta}\|f\|_{2, E},
$$

then

$$
\|S f\|_{p_{0}^{\prime} 2} \leq C|E|^{\theta}\|f\|_{2, E} .
$$

By scaling, this shows (ii) of Lemma (2.7). 


\section{Proof of Theorem 1}

We first make a reduction. Set $e_{d}=(0, \cdots, 0,1)$.

Lemma 3.1. To show Theorem 1, it is sufficient to show that for any given $\lambda>0$ there is $\epsilon>0$ such that if (3.2)-(3.6) below holds then $u=0$, where

$$
\begin{aligned}
& \Omega=R^{d} \overline{\backslash D\left(-e_{d}, \frac{1}{2}\right)}, u: \Omega \rightarrow R \\
& \operatorname{supp} u \subset \overline{D\left(-e_{d}, 1\right)}, 0 \in \operatorname{supp} u \\
& u \in W_{\mathrm{loc}}^{22}(\Omega) \\
& |L u| \leq A|u|+B|\nabla u|,
\end{aligned}
$$

where

$$
\begin{array}{r}
L=\sum_{i, j=1}^{d} a_{i j}(x) \frac{d^{2}}{d x_{i} d x_{j}} \\
\lambda|\xi|^{2} \leq \sum_{i, j=1}^{d} a_{i j}(x) \xi_{i} \xi_{j} \leq \lambda^{-1}|\xi|^{2},|| a_{i j} \|_{\Omega} \leq \epsilon
\end{array}
$$

where

$$
\begin{aligned}
& \left\|a_{i j}\right\|_{\Omega}=\sup _{x, y \in \Omega} \frac{\left|a_{i j}(x)-a_{i j}(y)\right|}{|x-y|} \\
& \|A\|_{L^{p \infty}(\Omega)} \leq \epsilon,\|B\|_{L^{d \infty}(\Omega)} \leq \epsilon
\end{aligned}
$$

where $p$ is the same as in Theorem 1.

Proof of Lemma 3.1 follows from a modification of the reductions in [9] and $[\mathbf{3}]$.

We now let $K$ be the convex hull of Supp $u \bigcap\left\{x_{d} \geq-1 / 4\right\}$. Select $\phi: R^{d} \rightarrow R$ such that $\phi(x)=0$ when $x_{d} \leq-1 / 3$ and $\phi=1$ on a neighborhood of $\partial K$. Set $v=\phi u$, then

$$
|L v| \leq A|v|+B|\nabla v|+\chi
$$


where $\chi \in L^{2}$ has the property:

$$
\operatorname{supp} \chi \subset A_{1} \bigcup A_{2}
$$

where $A_{1}=\overline{D\left(-e_{d}, 1\right.} \bigcap\left\{x:-1 / 3 \leq x_{d} \leq-1 / 4\right\}, A_{2}=$ a compact subset of Int $K$.

Let $\Gamma$ be the cone $\left\{k \in R^{d}: k_{d} \geq 4 \sqrt{|k|^{2}-k_{d}^{2}}\right\}$.

We now let $\epsilon>0$ in Lemma (3.1) be small, $M$ large and define

$$
\mu=\left(A|v|+B|\nabla v|+\epsilon M^{-1 / 2}\left|H_{v}\right|\right)^{2} d x
$$

By Lemma 1' (instead of Lemma 1) in [9], taking $p=2, \mathcal{C}=D$ $\left(2 M e_{d}, \frac{2 M}{100}\right)$, then we can select $\left\{k_{j}\right\}$ and $\left\{E_{j}\right\}$ with $\left\{E_{j}\right\}$ disjoint, compact and convex sets such that

$$
\begin{gathered}
\frac{M}{2} \leq\left|k_{j}\right| \leq 2 M, k_{j} \in \Gamma \\
\| e^{k_{j} \cdot x}\left(A|v|+B|\nabla v|+\epsilon M^{-1 / 2}\left|H_{v}\right|\right) \mid L_{L^{2}\left(R^{d} \backslash(1+T) E_{j}\right)} \\
\leq 2^{-1 / 2} e^{-1 / 2 C^{-1} T}|| e^{k_{j} \cdot x}\left(A|v|+B|\nabla v|+\epsilon M^{-1 / 2}\left|H_{v}\right|\right) \|_{2},
\end{gathered}
$$

provided $T \geq 0$, and

$$
\sum\left|E_{j}\right|^{-1} \geq C^{-1} M^{d}
$$

$$
\operatorname{diam} E_{j} \leq C M^{-1 / 2}
$$

Because the family of weights $\left\{e^{k \cdot x}\right\}$ is invariant by linear coordinate changes, we note the Carleman inequalities in Lemmas (2.1) and (2.7) remain valid if the Laplacian is replaced by any other constant coefficient second order elliptic operator, with bounds depending on ellipticity. We now let $L_{j}$ be the constant coefficient operator obtained by freezing the coefficients of $L$ at the barycenter of $E_{j}$.

Besides (3.0)-(3.13), we furthermore have the following 
Lemma 3.14. Under the assumptions (3.2)-(3.7), we can select $\left\{k_{j}\right\}$ and disjoint, compact and convex sets $\left\{E_{j}\right\}$ satisfying (3.11)-(3.15) and also the following inequalities (3.15)-(3.18) for any $\theta_{1}>\theta_{d}=\max \left(\frac{d-4}{d(d-1)}, 0\right)$, and $\theta_{2}>\frac{d-2}{d(d-1)}$.

(3.15) $\|A\|_{L^{p \infty}\left(E_{j}\right)}\left(M^{d}\left|E_{j}\right|\right)^{\theta_{1}}+\|B\|_{L^{d \infty}\left(E_{j}\right)}\left(M^{d}\left|E_{j}\right|\right)^{\theta_{2}} \geq C_{1}$

(3.16) $\|A\|_{L^{t}\left(E_{j}\right)} M^{\frac{d}{t}-2}\left(M^{d}\left|E_{j}\right|\right)^{\theta_{1}}+\|B\|_{L^{s}\left(E_{j}\right)} M^{\frac{d}{s}-1}\left(M^{d}\left|E_{j}\right|\right)^{\theta_{2}} \geq C_{2}$

(3.17) $\|A\|_{L^{t}\left(E_{j}\right)} M^{\frac{d}{t}-2}\left(M^{d}\left|E_{j}\right|\right)^{\theta_{1}}+\|B\|_{L^{d \infty}\left(E_{j}\right)}\left(M^{d}\left|E_{j}\right|\right)^{\theta_{2}} \geq C_{3}$

(3.18) $\|A\|_{L^{p \infty}\left(E_{j}\right)}\left(M^{d}\left|E_{j}\right|\right)^{\theta_{1}}+\|B\|_{L^{s}\left(E_{j}\right)} M^{\frac{d}{s}-1}\left(M^{d}\left|E_{j}\right|\right)^{\theta_{2}} \geq C_{4}$

provided that $t<p$ is very close to $p$ and $s<d$.

Proof: To show (3.15), we will apply Lemmas (2.1) (ii) and (2.7) (ii) and Lemmas (8.1) and (8.2) in [9] together with the properties (3.9)(3.13) of the sets $\left\{E_{j}\right\}$ and $k_{j}$. We also note below that $\frac{1}{2}-\frac{1}{q}=\frac{1}{p}, p$ is as in Theorem 1 , and $p_{0}^{\prime}=\frac{2 d}{d-2}$.

$$
\begin{aligned}
& \left\|e^{k_{j} \cdot x}\left(A|v|+B|\nabla v|+\epsilon M^{-1 / 2}\left|H_{v}\right|\right)\right\|_{L^{2}\left(E_{j}\right)} \\
\leq & \|A\|_{L^{p \infty}\left(E_{j}\right)}|| e^{k_{j} \cdot x} v\left\|_{L^{q 2}\left(E_{j}\right)}+\right\| B\left\|_{L^{d \infty}\left(E_{j}\right)}\right\| e^{k_{j} \cdot x} \nabla v \|_{L^{p_{0}^{\prime} 2}\left(E_{j}\right)} \\
+ & \epsilon M^{-1 / 2}\left\|e^{k_{j} \cdot x} H_{v}\right\|_{L^{2}\left(E_{j}\right)} \\
\preceq & \|A\|_{L^{p \infty}\left(E_{j}\right)}\left(M^{d}\left|E_{j}\right|\right)^{\theta_{1}}\left\|e^{k_{j} \cdot x} L_{j} v\right\|_{2, E_{j}} \\
+ & \|B\|_{L^{d \infty}\left(E_{j}\right)}\left(M^{d}\left|E_{j}\right|\right)^{\theta_{2}}\left\|e^{k_{j} \cdot x} L_{j} v\right\|_{2, E_{j}}+\epsilon\left\|e^{k_{j} \cdot x} L_{j} v\right\|_{2, E_{j}} .
\end{aligned}
$$

The last inequality follows from Lemmas (2.1) and (2.7) for $L_{j}$ and by using (3.12) after using Lemma (8.1) in [9]. On the other hand, by (3.6) and (3.12), we have

$$
\left|L_{j} v\right| \leq|L v|+C \epsilon M^{-\frac{1}{2}} g_{E_{j}}\left|H_{v}\right|
$$

Hence

$$
\left|L_{j} v\right| \leq A|v|+B|\nabla v|+C \epsilon M^{-\frac{1}{2}} g_{E_{j}}\left|H_{v}\right|+\chi
$$

Therefore, by dropping $\chi$ in view of Lemma (8.2) in [9] and using the exponential decay property $(3.10),(3.19)$ is bounded by

$$
\begin{aligned}
{\left[\|A\|_{L^{p \infty}\left(E_{j}\right)}\left(M^{d}\left|E_{j}\right|\right)^{\theta_{1}}+\|B\|_{L^{d \infty}\left(E_{j}\right)}\left(M^{d}\left|E_{j}\right|\right)^{\theta_{2}}+\epsilon\right] } & \\
\cdot & || e^{k_{j} \cdot x}\left(A|v|+B|\nabla v|+C \epsilon M^{-\frac{1}{2}} g_{E_{j}}\left|H_{v}\right|\right) \|_{L^{2}\left(E_{j}\right)}
\end{aligned}
$$


Hence we get by cancellation

$$
\|A\|_{L^{p \infty}\left(E_{j}\right)}\left(M^{d}\left|E_{j}\right|\right)^{\theta_{1}}+\|B\|_{L^{d \infty}\left(E_{j}\right.}\left(M^{d}\left|E_{j}\right|\right)^{\theta_{2}}+\epsilon \geq C_{1} .
$$

Since $\epsilon$ is assumed to be arbitrarily small, we get

$$
\|A\|_{L^{p \infty}\left(E_{j}\right)}\left(M^{d}\left|E_{j}\right|\right)^{\theta_{1}}+\|B\|_{L^{d \infty}\left(E_{j}\right)}\left(M^{d}\left|E_{j}\right|\right)^{\theta_{2}} \geq C_{1} .
$$

This shows (3.15).

We now recall the definitions of the operators $S_{1}, S_{2}, T_{1}, T_{2}$ defined in section 2 and $p$ is as in the statement of Theorem 1, and temporarily denote $\frac{1}{2}-\frac{1}{q}=\frac{1}{p}, \frac{1}{2}-\frac{1}{q_{1}}=\frac{1}{t}>\frac{1}{p}$, and $\frac{1}{2}-\frac{1}{q_{2}}=\frac{1}{s}>\frac{1}{d}$. We then apply Lemmas (2.1) (i) and (2.7) (i), Lemmas (8.1) and (8.2) in [9], and also (2.2) and (2.8).

$$
\begin{aligned}
& \left\|e^{k_{j} \cdot x}\left(A|v|+B|\nabla v|+\epsilon M^{-1 / 2}\left|H_{v}\right|\right)\right\|_{L^{2}\left(E_{j}\right)} \\
\leq & \left\|A T_{1}\left(e^{k_{j} \cdot x} L_{j} v\right)|+| B S_{1}\left(e^{k_{j} \cdot x} L_{j} v\right)\right\|_{L^{2}\left(E_{j}\right)} \\
+ & \left\|A T_{2}\left(e^{k_{j} \cdot x} L_{j} v\right)\right\|_{L^{2}\left(E_{j}\right)}+\left\|B S_{2}\left(e^{k_{j} \cdot x} L_{j} v\right)\right\|_{L^{2}\left(E_{j}\right)} \\
+ & \left\|\epsilon M^{-1 / 2} e^{k_{j} \cdot x} H_{v}\right\|_{L^{2}\left(E_{j}\right)} \\
\leq & \|A\|_{L^{p \infty}\left(E_{j}\right)}|| T_{1}\left(e^{k_{j} \cdot x} L_{j} v\right)\left\|_{q}+\right\| B\left\|_{L^{d \infty}}\right\| S_{1}\left(e^{k_{j} \cdot x} L_{j} v\right) \|_{p_{0}^{\prime} 2} \\
+ & \|A\|_{L^{t}\left(E_{j}\right)}|| T_{2}\left(e^{k_{j} \cdot x} L_{j} v\right)\left\|_{L^{q_{1}}\left(E_{j}\right)}+\right\| B\left\|_{L^{s}\left(E_{j}\right)}|| S_{2}\left(e^{k_{j} \cdot x} L_{j} v\right)\right\|_{L^{q_{2}}\left(E_{j}\right)} \\
+ & \epsilon\left\|e^{k_{j} \cdot x} L_{j} v\right\|_{2, E_{j}} \\
\preceq & \|A\|_{L^{p \infty}\left(E_{j}\right)}|| e^{k_{j} \cdot x} L_{j} v\left\|_{L^{2}}+\right\| B\left\|_{L^{d \infty}\left(E_{j}\right)}\right\| e^{k_{j} \cdot x} L_{j} v \|_{L^{2}} \\
+ & \|A\|_{L^{t}\left(E_{j}\right)} M^{\frac{d}{t}-2}\left(M^{d}\left|E_{j}\right|\right)^{\theta_{1}}\left\|e^{k_{j} \cdot x} L_{j} v\right\|_{2, E_{j}} \\
+ & \|B\|_{L^{s}\left(E_{j}\right)} \mid\left\|e^{k_{j} \cdot x} L_{j} v\right\|_{2, E_{j}} M^{\frac{d}{s}-1}\left(M^{d}\left|E_{j}\right|\right)^{\theta_{2}}+\epsilon\left\|e^{k_{j} \cdot x} L_{j} v\right\|_{2, E_{j}} \\
\preceq & {\left[\|A\|_{L^{p \infty}\left(E_{j}\right)}+\|B\|_{L^{d \infty}\left(E_{j}\right)}+\|A\|_{L^{t}\left(E_{j}\right)} M^{\frac{d}{t}-2}\left(M^{d}\left|E_{j}\right|\right)^{\theta_{1}}\right.} \\
+ & \left.\|B\|_{L^{s}\left(E_{j}\right)} M^{\frac{d}{s}-1}\left(M^{d}\left|E_{j}\right|\right)^{\theta_{2}}+\epsilon\right]\left\|e^{k_{j} \cdot x} L_{j} v\right\|_{2, E_{j}} \\
\preceq & {\left[\|A\|_{L^{p \infty}\left(E_{j}\right)}+\|B\|_{L^{d \infty}\left(E_{j}\right)}+\|A\|_{L^{t}\left(E_{j}\right)} M^{\frac{d}{t}-2}\left(M^{d}\left|E_{j}\right|\right)^{\theta_{1}}\right.} \\
+ & \left.\|B\|_{L^{s}\left(E_{j}\right)} M^{\frac{d}{s}-1}\left(M^{d}\left|E_{j}\right|\right)^{\theta_{1}}+\epsilon\right] \\
+ & \left\|e^{k_{j} \cdot x}\left(A|v|+B|\nabla v|+C \epsilon M^{-\frac{1}{2}} g_{E_{j}}\left|H_{v}\right|\right)\right\|_{L^{2}\left(E_{j}\right)} \cdot
\end{aligned}
$$

Thus by cancellation, we get

$$
\begin{aligned}
\|A\|_{L^{p \infty}\left(E_{j}\right)}+\|B\|_{L^{d \infty}\left(E_{j}\right)} & +\|A\|_{L^{t}\left(E_{j}\right)} M^{\frac{d}{t}-2}\left(M^{d}\left|E_{j}\right|\right)^{\theta_{1}} \\
& +\|B\|_{L^{s}\left(E_{j}\right)} M^{\frac{d}{s}-1}\left(M^{d}\left|E_{j}\right|\right)^{\theta_{2}}+\epsilon \geq C_{2} .
\end{aligned}
$$


Since $\|A\|_{L^{p \infty}\left(E_{j}\right)}$ and $\|B\|_{L^{d \infty}\left(E_{j}\right)}$ and $\epsilon$ are very small by assumption (see (3.7) in Lemma (3.1)), we may drop them and get

$$
\|A\|_{L^{t}\left(E_{j}\right)} M^{\frac{d}{t}-2}\left(M^{d}\left|E_{j}\right|\right)^{\theta_{1}}+\|B\|_{L^{s}\left(E_{j}\right)} M^{\frac{d}{s}-1}\left(M^{d}\left|E_{j}\right|\right)^{\theta_{2}} \geq C_{2} .
$$

This shows (3.16).

We now prove (3.17). We keep the same notation of $p, q$, and $q_{1}$ as in the proof of (3.16) above.

$$
\begin{aligned}
& \left\|e^{k_{j} \cdot x}\left(A|v|+B|\nabla v|+\epsilon M^{-1 / 2}\left|H_{v}\right|\right)\right\|_{L^{2}\left(E_{j}\right)} \\
\leq & \| A\left|T_{1}\left(e^{k_{j} \cdot x} L_{j} v\right)\right|+A\left|T_{2}\left(e^{k_{j} \cdot x} L_{j} v\right)\right| \\
+ & B\left|e^{k_{j} \cdot x} \nabla v\right|+\left.\epsilon M^{-1 / 2}\left|H_{v}\right|\right|_{L^{2}\left(E_{j}\right)} \\
\leq & \|A\|_{L^{p \infty}\left(E_{j}\right)}|| T_{1}\left(e^{k_{j} \cdot x} L_{j} v\right)\left\|_{L^{q}\left(E_{j}\right)}+\right\| A\left\|_{L^{t}\left(E_{j}\right)}\right\| T_{2}\left(e^{k_{j} \cdot x} L_{j} v\right) \|_{L^{q_{1}}\left(E_{j}\right)} \\
+ & \|B\|_{L^{d \infty}\left(E_{j}\right)}|| e^{k_{j} \cdot x} \nabla v\left\|_{L^{p_{0}^{\prime}{ }^{2}\left(E_{j}\right)}}+\epsilon\right\| e^{k_{j} \cdot x} L_{j} v \|_{2, E_{j}} \\
\preceq & \|A\|_{L^{p \infty}}|| e^{k_{j} \cdot x} L_{j} v\left\|_{2, E_{j}}+\right\| A\left\|_{L^{t}\left(E_{j}\right)} M^{\frac{d}{t}-1}\left(M^{d}\left|E_{j}\right|\right)^{\theta_{1}}|| e^{k_{j} \cdot x} L_{j} v\right\|_{2, E_{j}} \\
+ & \|B\|_{L^{d \infty}\left(E_{j}\right)}\left(M^{d}\left|E_{j}\right|\right)^{\theta_{2}}\left\|e^{k_{j} \cdot x} L_{j} v\right\|_{2, E_{j}}+\epsilon\left\|e^{k_{j} \cdot x} L_{j} v\right\|_{2, E_{j}} \\
\preceq & {\left[\|A\|_{L^{p \infty}\left(E_{j}\right)}+\left.\|A\|\right|_{L^{r}\left(E_{j}\right)} M^{d / r-1}\left(M^{d}\left|E_{j}\right|\right)^{\theta_{1}}\right.} \\
+ & \|B\|_{L^{d \infty}\left(E_{j}\right)}\left(M^{d}\left|E_{j}\right|\right)^{\theta_{2}} \\
+ & \epsilon] \cdot\left\|e^{k_{j} \cdot x}\left(A|v|+B|\nabla v|+C \epsilon M^{-\frac{1}{2}} g_{E_{j}}\left|H_{v}\right|\right)\right\|_{L^{2}\left(E_{j}\right)} .
\end{aligned}
$$

By cancellation, we get

$$
\begin{aligned}
\|A\|_{L^{p \infty}\left(E_{j}\right)}+\|A\|_{L^{t}\left(E_{j}\right)} M^{\frac{d}{t}-1}( & \left.M^{d}\left|E_{j}\right|\right)^{\theta_{1}} \\
& +\|B\|_{L^{d \infty}\left(E_{j}\right)}\left(M^{d}\left|E_{j}\right|\right)^{\theta_{2}}+\epsilon \geq C_{3} .
\end{aligned}
$$

By dropping $\|A\|_{L^{p \infty}\left(E_{j}\right)}$ and $\epsilon$ since they are very small, we get

$$
\|A\|_{L^{t}\left(E_{j}\right)} M^{\frac{d}{t}-2}\left(M^{d}\left|E_{j}\right|\right)^{\theta_{1}}+\|B\|_{L^{d \infty}\left(E_{j}\right)}\left(M^{d}\left|E_{j}\right|\right)^{\theta_{2}} \geq C_{3}
$$

this is (3.17)

Finally, we prove (3.18). We argue as follows:

$$
\begin{aligned}
& \| e^{k_{j} \cdot x}\left(A|v|+B|\nabla v|+\epsilon M^{-1 / 2} \mid H_{v} \|_{L^{2}\left(E_{j}\right)}\right. \\
& \leq\|A\|_{L^{p \infty}\left(E_{j}\right)}\left\|e^{k_{j} \cdot x} v\right\|_{L^{q^{2}}\left(E_{j}\right)}+\left\|B S_{1}\left(e^{k_{j} \cdot x} L_{j} v\right)\right\|_{L^{2}\left(E_{j}\right)} \\
& +\left\|B S_{2}\left(e^{k_{j} \cdot x} L_{j} v\right)\right\|_{L^{2}\left(E_{j}\right)}+\epsilon\left\|e^{k_{j} \cdot x} L_{j} v\right\|_{2, E_{j}} \\
& \preceq\|A\|_{L^{p \infty}\left(E_{j}\right)}\left(M^{d}\left|E_{j}\right|\right)^{\theta_{1}}\left\|e^{k_{j} \cdot x} L_{j} v\right\|_{2, E_{j}}+\|B\|_{L^{d \infty}\left(E_{j}\right)}\left\|e^{k_{j} \cdot x} L_{j} v\right\|_{L^{2}} \\
& +\|B\|_{L^{s}\left(E_{j}\right)}\left(M^{d}\left|E_{j}\right|\right)^{\theta_{2}} M^{\frac{d}{s}-1}\left\|e^{k_{j} \cdot x} L_{j} v\right\|_{2, E_{j}}+\epsilon\left\|e^{k_{j} \cdot x} L_{j} v\right\|_{2, E_{j}} \\
& \preceq\left[\|A\|_{L^{p \infty}\left(E_{j}\right)}\left(M^{d}\left|E_{j}\right|\right)^{\theta_{1}}\right. \\
& +\|B\|_{L^{d \infty}\left(E_{j}\right)}+\|B\|_{L^{s}\left(E_{j}\right)}\left(M^{d}\left|E_{j}\right|\right)^{\theta_{2}} M^{\frac{d}{s}-1} \\
& +\epsilon] \cdot|| e^{k_{j} \cdot x}\left(A|v|+B|\nabla v|+C \epsilon M^{-\frac{1}{2}} g_{E_{j}}\left|H_{v}\right|\right) \|_{L^{2}\left(E_{j}\right)} \text {. }
\end{aligned}
$$


By cancellation again, we get

$$
\begin{aligned}
\|A\|_{L^{p \infty}\left(E_{j}\right)} & \left(M^{d}\left|E_{j}\right|\right)^{\theta_{1}} \\
& +\|B\|_{L^{d \infty}\left(E_{j}\right)}+\|B\|_{L^{s}\left(E_{j}\right)}\left(M^{d}\left|E_{j}\right|\right)^{\theta_{2}} M^{\frac{d}{s}-1}+\epsilon \geq C_{4} .
\end{aligned}
$$

By dropping $\epsilon$ and $\|B\|_{L^{d \infty}\left(E_{j}\right)}$ we get

$$
\|A\|_{L^{p \infty}\left(E_{j}\right)}\left(M^{d}\left|E_{j}\right|\right)^{\theta_{1}}+\|B\|_{L^{s}\left(E_{j}\right)}\left(M^{d}\left|E_{j}\right|\right)^{\theta_{2}} M^{\frac{d}{s}-1} \geq C_{4} .
$$

this shows (3.18).

Lemma 3.20. Under the assumptions (3.2)-(3.7), we can either select a sequence of $\left\{E_{j}\right\}$ satisfying (3.9)-(3.13) such that (3.21) and (3.22) below hold:

$$
\begin{aligned}
& \|A\|_{L^{p \infty}\left(E_{j}\right)} \geq C^{-1}\left(M^{d}\left|E_{j}\right|\right)^{-\theta_{1}} \\
& \|A\|_{L^{t}\left(E_{j}\right)} \geq C^{-1}\left(M^{d}\left|E_{j}\right|\right)^{-\theta_{2}} M^{2-\frac{d}{t}}
\end{aligned}
$$

for any $\theta_{1}>\theta_{d}=\max \left(\frac{d-4}{d(d-1)}, 0\right)$ provided $t<p$ and very close to $p$; or a sequence of $\left\{E_{j}\right\}$ satisfying (3.9)-(3.13) but such that (3.23) and (3.24) below hold:

$$
\begin{aligned}
& \|B\|_{L^{d \infty}\left(E_{j}\right)} \geq C^{-1}\left(M^{d}\left|E_{j}\right|\right)^{-\theta_{2}} \\
& \|B\|_{L^{s}\left(E_{j}\right)} \geq C^{-1}\left(M^{d}\left|E_{j}\right|\right)^{-\theta_{2}} M^{-\frac{d}{s}+1}
\end{aligned}
$$

for any $\theta_{2}>\frac{d-2}{d(d-1)}$ provided $s<d$.

Proof: Take $C=\min \left\{C_{1}, C_{2}, C_{3}, C_{4}\right\}$ where $C_{1}, C_{2}, C_{3}$ and $C_{4}$ are constants in (3.15)-(3.18).

Given any $j$, if

$$
\|A\|_{L^{t}\left(E_{j}\right)}\left(M^{d}\left|E_{j}\right|\right)^{\theta_{1}} M^{\frac{d}{t}-2} \leq C / 2
$$

then (3.23) and (3.24) follow from this and (3.16) and (3.17).

If

$$
\|A\|_{L^{t}\left(E_{j}\right)}\left(M^{d}\left|E_{j}\right|\right)^{\theta_{1}} M^{\frac{d}{t}-2}>C / 2
$$

then we consider (3.15) and (3.18). If

$$
\|A\|_{L^{p \infty}\left(E_{j}\right)}\left(M^{d}\left|E_{j}\right|\right)^{\theta_{1}}>C / 2
$$

then combining (3.25) and (3.26) we will get (3.21) and (3.22). If

$$
\|A\|_{L^{p \infty}\left(E_{j}\right)}\left(M^{d}\left|E_{j}\right|\right)^{\theta_{1}} \leq C / 2
$$

then by (3.27), (3.15) and (3.18), we will get (3.23) and (3.24). 
Lemma 3.28. Under the assumptions (3.2)-(3.7), we can either select a sequence of $\left\{k_{j}\right\}$ and disjoint compact convex sets of $\left\{E_{j}\right\}$ satisfying (3.9)-(3.13) and there exist $\theta_{11}, \theta_{12}$ with $\theta_{d}<\theta_{11}, \theta_{12}<\frac{1}{d}$ and $t<p$ such that for each such $j$ there exists some $\lambda_{j}$ satisfying

$$
\left|\left\{x \in E_{j} ;|A(x)|>\lambda_{j}\right\}\right| \geq C_{\theta_{11}}^{-1} \lambda_{j}^{-p}\left(M^{d}\left|E_{j}\right|\right)^{-p \theta_{11}}
$$

and

$$
C^{-1}\left(M^{d}\left|E_{j}\right|\right)^{\frac{-\theta_{11} p-1}{p}} M^{\frac{d}{p}} \leq \lambda_{j} \leq C\left(M^{d}\left|E_{j}\right|\right)^{\frac{\theta_{12} t}{p-t}} M^{\frac{d}{p}}
$$

or select a sequence of $\left\{k_{j}\right\}$ and $\left\{E_{j}\right\}$ satisfying (3.9)-(3.13) and there exist $\theta_{21}, \theta_{22}$ with $\frac{d-2}{d(d-1)}<\theta_{21}, \theta_{22}<\frac{1}{d}$ and $s<d$, such that for each such $j$ there exists some $\lambda_{j}$ satisfying (3.31)-(3.32) below:

$$
\begin{aligned}
& \left|\left\{x \in E_{j}:|B(x)|>\lambda_{j}\right\}\right| \geq C_{\theta_{21}}^{-1} \lambda_{j}^{-d}\left(M^{d}\left|E_{j}\right|\right)^{-d \theta_{21}} \\
& C^{-1}\left(M^{d}\left|E_{j}\right|\right)^{\frac{-\theta_{21} d-1}{d}} M \leq \lambda_{j} \leq C\left(M^{d}\left|E_{j}\right|\right)^{\frac{\theta_{22} s}{d-s}} M
\end{aligned}
$$

We note here that if (3.23) and (3.24) in Lemma (3.20) are true, then (3.31) and (3.32) follow as shown in [3]. We can also show that if (3.21) and (3.22) in Lemma (3.20) hold, then (3.29) and (3.30) also hold. Since the proof is quite similar, we omit the details.

By Lemma (3.28) and the combinatorial lemma proved in [3], we will get the proof of Theorem 1. We omit the details here.

\section{References}

[1] S. Chanillo and E. Sawyer, Unique Continuation for $\Delta+V$ and the C. Fefferman-Phong class, Trans. Amer. Math. Soc. 318 (1990), 275-300.

[2] D. Jerison and C. Kenig, Unique continuation and absence of positive eigenvalues for Schrödinger operators, Ann. Math. 12 (1985), 463-492.

[3] G. Lu AND T. WolfF, Unique continuation with weak type lower order terms, to appear.

[4] A. Plis, On nonuniqueness in Cauchy problems for an elliptic second order differential operator, Bull. Acad. Polon. Sci. 11 (1963), $95-100$. 
[5] A. Ruiz And L. VEGA, Unique continuation for the solutions of the Laplacian plus a drift, Ann. Institute Grenoble 41(3) (1991), 651-663.

[6] E. Stein, Appendix to "Unique continuation", Ann. Math. 121 (1985), 492-494.

[7] C. Sogge, Oscillatory integrals and unique continuation for second order differential equations, J. Amer. Math. Soc. 2 (1989), 489-515.

[8] C. Sogge, Strong uniqueness theorems for second order elliptic differential equations, Amer. J. Math. 112 (1990), 943-984.

[9] T. WolfF, A property of measures in $R^{N}$ and an application to unique continuation, Geometric and Functional Analysis 2(2) (1992), 225-284.

[10] T. WolfF, Unique continuation for $|\triangle u| \leq V|\nabla u|$ and related problems, Revista Mathematica Iberoamericana 6(3-4) (1990), 155-200.

Department of Mathematics and Statistics

Wright State University

Dayton, OH 45435

U.S.A.

Rebut el 23 de Desembre de 1994 